withdrawal, acute severe ulcerative colitis, isolated proctitis, or performance as part of a trial were features deemed inappropriate for IUS. In either indication, obesity (BMI>30) or complex surgical history were considered unsuitable for IUS.

Results 260 endoscopies were performed for the specified indications, of which $73(28.1 \%)$ met criteria to be performed as IUS instead (55 of 220 colonoscopies [25\%] \& 18 of 40 flexible sigmoidoscopies [45\%]). By indication, 46 of 108 [42.6\%] cases performed for IBD assessment \& 27 of 152 [17.8\%] for symptoms met criteria.

Among potential IUS patients, one case each of adenoma $(<5 \mathrm{~mm}) \&$ sessile serrated lesion $(<5 \mathrm{~mm})$ were found; no other significant pathology that would be expected to be missed with IUS was encountered. Further, there were no cases of isolated proctitis found among IBD patients deemed suitable for IUS. In contrast, among IUS unsuitable patients, 4 malignancies, 19 cases of adenomatous polyps (6 cases of 5$10 \mathrm{~mm} \& 1$ case of $>10 \mathrm{~mm}), \& 8$ new cases of IBD were diagnosed, supporting the proposed criteria.

Conclusion Using conservative criteria, almost 30\% of endoscopies for IBD assessment or symptoms could have been performed as IUS instead, with a potential annual cost saving of $>£ 400,000$. No cases of significant non-inflammatory pathology would have been missed with IUS using the proposed criteria.

\section{PM0-27 SWITCHING TO SUBCUTANEOUS VEDOLIZUMAB IN INFLAMMATORY BOWEL DISEASE DURING COVID-19 PANDEMIC - COUNTY DURHAM EXPERIENCE}

\footnotetext{
1,2 2 rances Maw, 'Mei Yen Chan, 'Susan Ritchie, 'Victoria Fletcher, 'Helen Dallal, ${ }^{1,3}$ Anjan Dhar*. 'Department of Gastroenterology, County Durham and Darlington Nhs Foundation Trust, Darlington, UK; ${ }^{2}$ Department of Clinical Pharmacy, Darlington Memorial Hospital, Darlington, UK; ${ }^{3}$ School of Health and Life Sciences, Teesside University, Middlesbrough, UK
}

\subsection{6/gutjpl-2021-BSG. 166}

Introduction The COVID-19 pandemic resulted in the need for significant adaptations to an intravenous biologics infusion service. Patients were reluctant to come to a Biologics Infusion Unit in an acute hospital due to the fear of exposure to SARS-CoV-2 virus and also due to the UK governmental advice on strict isolation and shielding. Based on the VISIBLE1 and -2 Study data, we decided to switch our IBD patients from IV Vedolizumab to SC vedolizumab in a phased manner. We report on our experience and short term outcomes.

Methods During the COVID-19 pandemic, we decided to switch patients with Ulcerative colitis and Crohn's disease from IV Vedolizumab to Subcutaneous Vedolizumab in a phased manner. In the first Phase (July - Sep 2020) all patients with Ulcerative colitis (UC) in clinical remission of their disease beyond 16 weeks of Vedolizumab treatment were invited to switch. In phase 2 (October -Dec 2020) additional UC and Crohn's Disease (CD) patients on maintenance were switched and in Phase 3, new patients with UC and CD entering the Vedolizumab treatment programme were included for IV induction followed by SC maintenance. 6 month outcomes were assessed for clinical response, faecal calprotectin, and for short term patient experience.

Results A total of 31 patients were switched in the 6 month period of COVID-19 adaptation. 19 patients had UC (13 pancolitis, 6 distal colitis) and 12 patients had CD (1 Crohn's colitis, 5 ileocolonic, 5 small bowel and 1 complex). Over the 6 month period, 1 patient flared on SC Vedo and switched back to escalated IV, 1 developed abnormal LFTs and switched out of class. All remaining patients were doing well, and had mean SCAI score of 0 , HBI score of 1 . Mean FCP in UC patients was $197 \mathrm{ug} / \mathrm{g}$ (range 25-561), and in CD patients was 248ug/g (range 213-283). Patient experience indicated that all patients on SC Vedo felt that they were safer during the pandemic.

Conclusion A COVID-19 adaptation of managed switching of patients with ulcerative colitis and Crohn's disease from IV to SC Vedolizumab is safe and does not result in any adverse outcomes. Long term data on maintenance of remission and endoscopic healing is awaited.

\section{PMO-28 EFFICACY OF SUBCUTANEOUS INFLIXIMAB IN PERIANAL CROHN'S DISEASE}

Philip J Smith*, Daniel Storey, Belle Gregg, Lisa Critchley, June Stenson, Ashley Bond, Alan Steel, Martyn Dibb, Paul Collins, Edmund Derbyshire, Christopher Probert, Sreedhar Subramanian. Royal Liverpool Hospital, Department of Gastroenterology, Liverpool University Hospitals Foundation NHS Trust, Liverpool, UK, Liverpool, UK

\subsection{6/gutjnl-2021-BSG.167}

Background A new subcutaneous (SC) formulation of infliximab has recently been approved for the management of inflammatory bowel diseases (IBD). In the registration clinical trial, the efficacy of SC IFX was comparable to intravenous (IV) IFX (1) but specific data regarding the efficacy of SC IFX in perianal Crohn's disease (pCD) is lacking. We aimed to investigate the efficacy of SC IFX in patients with pCD who were switched from IV IFX.

Methods We conducted a single centre observational, retrospective study of pCD patients who were maintained on IV IFX and switched to SC IFX. Elective switching was at the discretion of the treating physician. In patients with active pCD at treatment initiation, the success of SC IFX was defined by clinical success at 6 months assessed by the physician's judgment without additional medical or surgical treatment for $\mathrm{pCD}$. In patients with inactive $\mathrm{pCD}$, the $\mathrm{pCD}$ recurrence-free survival was calculated. Treatment persistence rates were calculated at the end of 6 months. Safety and adverse events of interest were recorded. Data was analysed using SPSS for Windows with $\mathrm{p}<0.05$ being significant.

Results We included 18 patients with a mean age of 36 (SD 12) and a M:F ratio of $5: 4$. Eleven (61\%) patients were on concomitant thiopurines with a mean 6-thioguanine level of 258 (SD 155). The majority $(n=13,72 \%)$ had an inter-sphincteric fistula, 1 patient had a supra-sphincteric fistula (5.6\%) and 2 patients $(11.1 \%)$ each had trans-sphincteric and extrasphincteric fistulae respectively. None of the patients had active, draining fistulae at the time of switching and 1 patient had an examination under anaesthesia (EUA) in the 6 weeks prior to switch. The mean IFX level at baseline was $9.9 \square \mathrm{g} /$ $\mathrm{ml}$ (SD 4.1). Two patients (11.1\%) had recurrence of symptoms after switching to SC IFX and required further antibiotic therapy and EUA. These two patients also switched back to IV IFX after a median of 2.25 months. The mean IFX levels at 3 and 6 months after switch were $15.6 \mathrm{Dg} / \mathrm{ml}$ (SD 0.2) and $15.5 \square \mathrm{g} / \mathrm{ml}(\mathrm{SD} 1.4)$ respectively $(\mathrm{P}<0.01)$. The treatment 